\title{
Non-specific interstitial pneumonia and features of connective tissue disease: What are the consequences of a different point of view?
}

\author{
Alice Biffi', Giulia Dei', Federica De Giacomi1, Anna Stainer'1, Lorenzo Olmo Parma², \\ Maria Rosa Pozzi², Paola Faverio', Alberto Pesci1 \\ 1 Dipartimento Cardio-Toraco-Vascolare, University of Milan Bicocca, Respiratory Unit, \\ San Gerardo Hospital, Monza \\ 2 Dipartimento di Medicina Interna, UOSD Reumatologica, San Gerardo Hospital, Monza, Italy
}

\begin{abstract}
Patients with Interstitial Lung Disease (ILD) without a definitive diagnosis of connective tissue diseases (CTD) were historically described as Undifferentiated Connective Tissue Disease (UCTD-ILD). Recently a new classification, Interstitial Pneumonia with Autoimmune Features (IPAF), has been proposed. Aim of this study was to describe the prevalence, clinical characteristics and prognostic factors of UCTD and IPAF subjects in a cohort of Non-Specific Interstitial Pneumonia (NSIP) patients. This retrospective observational study enrolled 102 adult patients characterized by NSIP pattern on High Resolution Computed Tomography, without a specific diagnosis of CTD. Three groups were identified according to patients' characteristics: IPAF, UCTD or idiopathic NSIP (iNSIP). Forty percent, $27 \%$ and $55 \%$ of patients showed diagnostic criteria for IPAF, UCTD and iNSIP, respectively. No significant differences in age, gender, smoking habit, pulmonary function tests and three-year survival rate were observed
\end{abstract}

Corresponding author: Federica De Giacomi, Dipartimento Cardio-ToracoVascolare, University of Milan Bicocca, Respiratory Unit, San Gerardo Hospital, via Pergolesi 33, 20900 Monza, Italy.

Tel: +39.333.5629379 - Fax: +39.039.2336660. E-mail: i.fede@live.it

Key words: Interstitial lung diseases; connective tissue diseases; nonspecific interstitial pneumonia; interstitial pneumonia with autoimmune features; antisynthetase syndrome.

Contributions: AB, GD, FDG, LOP, data acquisition; AB, GD, FDG, AS, LOP, PF, manuscript drafting; MRP, AP, critical revision of the manuscript for important intellectual content; Read and approval of the final manuscript. All authors conceived and designed the study, and approved the final version of the manuscript.

Conflict of interest: The authors declare no conflict of interest.

Received for publication: 27 May 2018

Accepted for publication: 21 August 2018

(C) Copyright A. Biffi et al., 2018

Tipografia PI-ME Editrice, Italy

Monaldi Archives for Chest Disease 2018; 88:970

doi: 10.4081/monaldi.2018.970

This article is distributed under the terms of the Creative Commons Attribution Noncommercial License (by-nc 4.0) which permits any noncommercial use, distribution, and reproduction in any medium provided the original author(s) and source are credited. among study groups. IPAF patients with antisynthetase antibodies positivity, in comparison to IPAF without antisynthetase antibodies positivity, showed more frequently an acute onset (44\% vs $9 \%, \mathrm{p}<0.012)$. The presence of autoimmune features seems not to be associated with better outcomes in NSIP patients. IPAF criteria seem to be more representative than UCTD criteria in identifying patients with autoimmune features. Further studies are needed to verify if IPAF should include patients with positive antisynthetase serology.

\section{Introduction}

Pulmonary involvement, particularly interstitial lung disease, is a significant complication of many Connective Tissue Diseases (CTD) and may precede the onset of the disease itself [1].

Up to $25 \%$ of patients with symptoms of connective tissue disease do not receive a definitive diagnosis of CTD, according to validated diagnostic criteria. These patients were historically described as having "collagen" or "unclassifiable connective tissue" diseases, and, since they shared similar clinical features of widespread inflammation, they have also been identified as "diffuse" or "Undifferentiated CTD" (UCTD) $[2,3]$.

The Non Specific Interstitial Pneumonia (NSIP) pattern of lung injury (both radiological and pathological) has been noted as common in patients who are classified as having UCTD [3]. This finding has important implications, since the prognosis and treatment response are more favourable in rheumatic disease-associated NSIP compared to idiopathic NSIP (iNSIP) [4].

The concept of "lung-dominant CTD" has been proposed to characterize those patients with NSIP who had certain histopathological features or positive serologies, but lacked sufficient extra-pulmonary clinical features to be classified as having a specific rheumatic disease [5].

Recently, the European Respiratory Society and American Thoracic Society proposed the term "interstitial pneumonia with autoimmune features (IPAF)" for patients with interstitial lung disease (ILD) and features of autoimmune diseases [6]. However, the effort to hyper-categorize different diseases with a number of diagnostic criteria may result in an artificial subclassification.

Aim of this study is to describe the prevalence of patients with ILDs and autoimmune features (not classified as CTD by validated diagnostic criteria), including both patients with UCTD and those with IPAF and to identify the clinical characteristics and prognostic factors of UCTD and IPAF subjects in a cohort of consecutive patients with NSIP pattern on High Resolution Computed Tomography (HRCT). Moreover, we aim to describe the characteristics of the subgroup of patients with positive antisynthetase serology, in order to assess if Antisynthetase Syndrome (ASS) may represent a different population. 


\section{Materials and Methods}

\section{Study population}

We retrospectively analysed a cohort of 195 consecutive patients, characterized by NSIP pattern on HRCT, referred to tertiary referral outpatient ILD Clinic of the San Gerardo Hospital, Monza, Italy from November 2006 to December 2016. The Institutional Review Board of our Institution approved the study (\#980, 31 August 2016) and informed consent was waived because of the retrospective nature of the study.

Radiological NSIP pattern was defined by an expert radiologist, blinded to any clinical, functional and laboratory data, as the presence of relatively symmetric and bilateral ground glass opacities, fine reticulations and/or traction bronchiectasis, lower lobe prevalence and absence of honeycombing [7]. If present, subpleural sparing was considered suggestive for NSIP [8].

The final diagnosis was achieved by multidisciplinary discussion involving pulmonologists, radiologists, rheumatologists and pathologists. IPAF diagnosis was based on the recent consensus statement [6], UCTD diagnosis was based on Kinder Criteria [3] and ASS diagnosis was based on Connors' criteria [9].

\section{Data collection}

We extracted data regarding demographics, smoking and exposure history, past medical history and clinical examination with particular attention to the presence of signs and symptoms suggestive of rheumatological diseases. Rheumatological signs or symptoms analyzed were selected prior to data extraction, based on IPAF and UCTD criteria $[3,6]$.

Autoimmunity test results [Anti-Nuclear Antibodies (ANA), Extractable Nuclear Antigens (ENA), anti-Cyclic Citrullinated Peptide antibodies (anti-CCP), Rheumatoid Factor (RF)], performed as part of the initial diagnostic work-up of uncharacterized ILD, were collected in all patients (see Table 1 for institutional ranges and cut-offs). From December 2014 anti-myositis antibodies enlarged panel was also determined in 46 subjects (Table 1).

Acute onset of respiratory symptoms was defined if symptoms developed within one month from the first medical visit. Pulmonary function tests (PFTs), including forced vital capacity (FVC), diffusing capacity for carbon monoxide (DLCO), six-minute walking test (6MWT) (distance walked and lower oxygen saturation measured) and oxygen supplementation requirement [if partial pressure of oxygen $(\mathrm{PaO2})$ at rest was $<55 \mathrm{mmHg}$ or lower pulse oximetry during the $6 \mathrm{MWT} \leq 88 \%$ ] were collected.

GAP index [10] was calculated GAP index was calculated in 91/102 patients at time of diagnosis, because the remaining 11 patients were not able to perform DLCO assessment.

HRCT scans were reviewed for the presence of emphysema, reticulations, ground glass opacities by an expert radiologist.

\section{Statistical analysis}

Statistical analysis was performed using SPSS® Statistics software (IBM®, ver. 21.0) on Windows platform. Categorical variables were expressed as absolute number $(\mathrm{N})$ and/or percentage (\%) and were compared with Fisher or chi-square test where appropriate. Continuous variables, expressed as median and interquartile range [IQR] were compared with Kruskal-Wallis test. Two-sided P values $<0.05$ were considered statistically significant. Kaplan-Maier method and log rank test were used for the survival analysis. Univariate Cox regression analysis was applied to identify prognostic factors.

\section{Results}

\section{Demographic and clinical characteristics}

Out of 195 patients presenting with radiological NSIP pattern, 31 were excluded because of a prior diagnosis of CTD using validated diagnostic criteria at the time of diagnosis or during follow-up, 43 and 19 subjects were excluded because of a prior diagnosis of chronic hypersensitivity pneumonitis and drug-induced ILD, respectively. The remaining 102 patients (median age 67 years, $51 \%$ males) without a specific diagnosis were included in the analysis. Forty-two (41\%) patients were never smokers, 11 (11\%) current smokers and 49 (48\%) former smokers. The main comorbidities registered were systemic hypertension (45\%), diabetes (21\%) and ischemic heart disease (17\% of patients). At baseline the median [IQR] FVC was $78(62-95) \%$ of predicted and DLCO 43 (32-60)\% of predicted. The median [IQR] meters walked during 6MWT were $321 \mathrm{~m}$ (215-385). At baseline, 39 (38\%) patients were classified as stage I of GAP index, 35 (34\%) as stage II and 17 (17\%) as stage III. The median [IQR] follow-up time was 43 months (22-62).

Forty-two percent of patients complained of dyspnoea at rest and/or under effort, $10 \%$ of cough and $38 \%$ of both dyspnoea and cough. Fifteen percent of patients experienced an acute onset of respiratory

Table 1. Autoimmune serology screening panel.

\begin{tabular}{|c|c|c|c|c|}
\hline Test name & Technique & Reference values & Screening positive cut-off & Positive $(\%)(\mathbb{N}=102)$ \\
\hline ANA, serum & Indirect immunofluorescence & $<1: 80$ (negative) & $\geq 1.80$ (titre and pattern were specified) & $29(28)$ \\
\hline SS-A/Ro antibody, serum IgG & Immunoblotting & $<1.0$ (negative) & $\geq 1.0$ (positive) & $10(10)$ \\
\hline SS-B/La antibody, serum IgG & Immunoblotting & $<1.0$ (negative) & $\geq 1.0$ (positive) & $2(2)$ \\
\hline Scl 70 antibody, serum & Immunoblotting & $<1.0$ (negative) & $\geq 1.0$ (positive) & $2(2)$ \\
\hline Jo-1 antibody, serum IgG & Immunoblotting & $<1.0$ (negative) & $\geq 1.0$ (positive) & $13(13)$ \\
\hline $\begin{array}{l}\text { RF, serum antibody } \\
\text { (all classes against Fc of IgG) }\end{array}$ & Immunoturbid & $0-14 \mathrm{IU} / \mathrm{mL}$ & $\geq 15 \mathrm{IU} / \mathrm{mL}$ & $14(14)^{*}$ \\
\hline CCP antibody, serum & ELISA & $<20.0 \mathrm{U}$ & $\geq 20 \mathrm{U}$ & $3(3)$ \\
\hline $\begin{array}{l}\text { Anti-myositis antibodies including } \\
\text { anti-Mi2, anti-Ku, anti-PM-Scl75, } \\
\text { anti-PM-Scl100, anti-SRP, anti-PL7, } \\
\text { anti-PL12, anti-EJ, anti-OJ }\end{array}$ & Immunoblotting & & & $6(6)$ \\
\hline
\end{tabular}


symptoms as first presentation of disease. Ten percent of patients were asymptomatic and were assessed because of accidental findings on chest X-ray.

Rheumatologic-related signs and symptoms, according to the IPAF [6] and UCTD criteria [3], were recorded in 48 patients (47\%) (Table 2).

The most common reported symptoms were gastroesophageal reflux (18\%) and arthralgia/multiple joint swelling (13\%). Distal digital fissuring ("mechanic hands") were present in $10 \%$ of patients, Raynaud's phenomenon in $7 \%$ of patients and unexplained digital oedema in $1 \%$ of patients.

At baseline, broncoalveolar lavage was performed in 73 (74\%) patients, transbronchial biopsies in 15 (15\%), and surgical lung biopsy in 6 (6\%) patients.

At 6-month follow up, 53 patients were treated with corticosteroids, 13 with steroid-sparing agents associated to steroids (11 with azathioprine and 2 with cyclophosphamide).

\section{Serologic assessment}

All 102 patients included were assessed through autoimmune serology panel (Table 1). Fifty-four patients (53\%) had a negative autoimmune serology panel.

ANA antibodies were positive in 29 patients (28\%) (with 1:80 title in 6 cases, 1:160 in 6, 1:320 in 7, and >1:320 in 10). Patients showed a homogeneous ANA pattern in 11 cases (38\%), speckled in 14 (48\%), nucleolar in 2 (7\%) and cytoplasmic in 2 (7\%). Out of the 17 patients with ANA titer $\geq 320,9$ showed a granular, 7 homogenous, and one nucleolar pattern. ENA antibodies were positive in 13 patients (13\%), particularly $\mathrm{SSA} / \mathrm{Ro}$ were positive in $10 \%$ of patients, $\mathrm{SSB} / \mathrm{La}$ and $\mathrm{Scl} 70$ in $2 \%$ of patients respectively. One patient had double ENA positivity (anti-SSA/Ro and anti-SSB/La). Eight ANA positive patients were also ENA positive (27.6\% of ANA positive patients).

Eighteen patients (18\%) were characterized by positive antisynthetase antibodies serologic testing.

Anti-Jol (anti-histidyl-tRNA synthetase) antibodies, tested in all patients, were positive in 13 cases (13\%). Among the 46 patients tested, anti-myositis antibodies enlarged panel was positive in 6 cases (13\%): anti PL7 (anti-threonyl-tRNA synthetase) were detected in 2 patients, and anti PL12 (anti-alanine-tRNA synthetase), anti OJ (anti-isoleucyltRNA synthetase), anti EJ (anti-glycyl-tRNA synthetase) and anti SRP (signal recognition particle) in one case each.

\section{Comparison between IPAF, UCTD, and iNSIP groups}

IPAF criteria were met in 41 patients (40\%), UCTD criteria in 28 (27\%), and, the remaining 56 patients (55\%) were classified as iNSIP. IPAF diagnosis was achieved as follows: 21 patients met both morphological (radiological NSIP HRCT pattern) and serological domains, 4 met both morphological (radiological NSIP HRCT pattern) and clinical domains, and 16 patients met all the three domains. Twenty-three (22\%) subjects had overlapping diagnosis of IPAF and UCTD, while 18 (18\%) and 5 (5\%) patients satisfied only IPAF and UCTD criteria, respectively. The clinical characteristics that allowed to classify 5 patients into UCTD only were gastroesophageal reflux in 4 cases and skin changes (rash) in one case. The autoimmune serological assessment of these 5 patients revealed ANA positivity (with title 1:80 in 2 cases and 1:160 in one case) and/or RF at low title (one patient had both ANA and RF positivity).

No significant differences in age, gender, smoking habit, PFTs or time of onset were observed among IPAF, UCTD and iNSIP groups (Table 3). A higher prevalence of respiratory failure at rest or under effort was observed in the IPAF group compared with the iNSIP group (22 vs 7\%, respectively, $\mathrm{p}=0.034$ ).

\section{Survival and prognostic factors}

The overall survival of the entire cohort at one, two and three years was $96 \%, 85 \%$ and $73 \%$, respectively. There was no difference in threeyear survival among IPAF, UCTD and iNSIP groups (IPAF 79\%, UCTD $78 \%$, iNSIP 71\%, p-value log rank 0.39). In the univariate analysis (Table 4), higher age at diagnosis (HR 1.055, 95\% CI 1.015-1.097, p-value 0.006) and higher GAP index (HR 2.811, 95\% CI 1.421-5.560, p-value 0.003 ) were directly correlated with overall mortality. FVC less

Table 2. Signs and symptoms UCTD and IPAF-related.

\begin{tabular}{|c|c|}
\hline Signs and symptoms & n (\%) of patients $(\mathrm{N}=102)$ \\
\hline Raynaud's phenomenon*\$ & $7(7)$ \\
\hline Arthralgia/multiple joint swelling* & $13(13)$ \\
\hline Photosensitivity* & $1(1)$ \\
\hline Unintentional weight loss ${ }^{*}$ & $8(8)$ \\
\hline Morning stiffness* & $6(6)$ \\
\hline Dry mouth or dry eyes (sicca features)* & $2(2)$ \\
\hline Dysphagia* & $3(3)$ \\
\hline Recurrent unexplained fever* & $2(2)$ \\
\hline Gastroesophageal reflux* & $18(18)$ \\
\hline Skin changes (rash) ${ }^{*}$ & $3(3)$ \\
\hline Oral ulceration* & $0(0)$ \\
\hline Nonandrogenic alopecia* & $0(0)$ \\
\hline Proximal muscle weakness** & $5(5)$ \\
\hline Distal digital fissuring (i.e. "mechanic hands")§ & $10(10)$ \\
\hline Distal digital tip ulceration§ & $0(0)$ \\
\hline Polyarticular morning joint stiffness $>60$ min*§ or inflammatory arthritis§ & $9(9)$ \\
\hline Palmar telangiectasia§ & $0(0)$ \\
\hline Unexplained digital oedema & $1(1)$ \\
\hline Unexplained fixed rash on the digital extensor surfaces (Gottron's sign)§ & $0(0)$ \\
\hline
\end{tabular}


than $60 \%$ of predicted (HR 2.278, 95\% CI 1.147-4,525, p-value 0.019), DLC0 less than $40 \%$ of predicted (HR 3.034, 95\% CI 1.311-7.025, p-value 0.010), shorter 6MWT distance walked (HR 0.995, 95\% CI 0.992-0.998, p-value 0.001 ) and lower oxygen saturation (HR 0.895, 95\% CI 0.8210.974 , p-value 0.011 ) at baseline were identified as negative prognostic factors.

\section{IPAF and antisynthetase antibodies syndrome}

In the IPAF group, all patients with positive antisynthetase antibody serologic testing were also classified as ASS, according to Connor's criteria [9]. Comparing the clinical characteristics of IPAF patients with positive antisynthetase antibodies (IPAF-ASS subgroup) ( $n=18,44 \%$ of all IPAF patients) to IPAF subjects with negative antisynthetase antibodies (IPAF-nonASS subgroup) ( $\mathrm{n}=23,56 \%$ of all IPAF patients), no significant differences were observed in regards to gender, age at diagnosis, smoking habit, baseline FVC\%, baseline DLCO\%, and oxygen requirement. IPAF-
ASS patients had more frequently an acute onset compared to other IPAF-nonASS patients (44\% vs $9 \%$, respectively, $\mathrm{p}<0.012$ ). During follow up, two IPAF-non-ASS patients experienced acute exacerbation, whereas no acute exacerbation was described in the IPAF-ASS group.

No differences in three-year survival between the IPAF-nonASS and the IPAF-ASS subgroups were observed ( $87 \%$ vs $62 \%$ respectively, log rank test 0.072 ), even if a worse trend was observed in IPAF-ASS (Figure 1).

\section{Discussion}

We described a real life cohort of 102 patients with NSIP HRCT pattern at presentation, $40 \%$ of them met the IPAF classification criteria, $27 \%$ the UCTD criteria, and $22 \%$ met both IPAF and UCTD criteria. To the best of our knowledge, this is the first study to analyse the fre-

Table 3. Clinical and functional characteristics and survival in the entire cohort and among different groups.

\begin{tabular}{|c|c|c|c|c|c|}
\hline Characteristics & Whole cohort $(\mathrm{N}=102)$ & IPAF $(\mathrm{N}=41)$ & UCTD ( $\mathrm{N}=28)$ & iNSIP $(\mathrm{N}=56)$ & p value \\
\hline Age at diagnosis (years), median [IQR] & $67[60-74]$ & $68[59-73]$ & $67[58-74]$ & $67[60-74]$ & 0.896 \\
\hline Age of first symptoms (years), median [IQR] & 66 [59-73] & $66[58-70]$ & $66[58-74]$ & 66 [59-73] & 0.853 \\
\hline Acute onset, N (\%) & $15(14 \%)$ & $10(24 \%)$ & $6(21 \%)$ & $5(9 \%)$ & 0.1 \\
\hline Sex (female), N (\%) & $50(49 \%)$ & $24(58 \%)$ & $18(64 \%)$ & $23(41 \%)$ & 0.079 \\
\hline Former or current smokers, N (\%) & $41(40 \%)$ & $21(51 \%)$ & $12(43 \%)$ & $38(68 \%)$ & 0.063 \\
\hline FVC \%, median [IQR] & 78 [62-95] & $81[63-97]$ & $73[58-90]$ & 75 [62-95] & 0.596 \\
\hline DLCO \%, median [IQR] & $43[32-60]$ & $44[33-53]$ & $40[31-52]$ & $42[29-64]$ & 0.951 \\
\hline Need of oxygen therapy, N (\%) & $13(13 \%)$ & $9(22 \%)$ & $5(18 \%)$ & $4(7 \%)$ & $\begin{array}{c}0.128 \\
\text { vs iNSIP } \mathrm{p}=0.034\end{array}$ \\
\hline Three years survival, N (\%) & $62(73 \%)^{*}$ & $19(79 \%)^{*}$ & $14(78 \%)^{\S}$ & $40(71 \%)$ & 0.39 \\
\hline
\end{tabular}

DLCO\%: percent predicted diffusing capacity for carbon monoxide; FVC\%: percent predicted forced vital capacity; IPAF: interstitial pneumonia with autoimmune features; iNSIP: idiopathic non-specific pneumonia; UCTD: undifferentiated connective tissue disease. *Missing data for 17 patients; §missing data for 10 patients.

Table 4. Univariate clinical predictors of mortality for the entire cohort.

\begin{tabular}{lll} 
Clinical and demographics characteristics & HR $(95 \%$ CI $)$ & \\
\hline Age at diagnosis & $1.055(1.015-1.097)$ & 0.006 \\
\hline Male sex & $1.243(0.900-1.717)$ & 0.186 \\
\hline Never smoker & $0.661(0.333-1.310)$ & 0.235 \\
Baseline PFTs & & \\
\hline FVC\% & $0.986(0.970-1.002)$ & 0.091 \\
\hline FVC $<60 \%$ & $2.278(1.147-4.525)$ & 0.019 \\
\hline DLCO\% & $0.969(0.949-0.990)$ & 0.004 \\
\hline DLCO <40\% & $3.034(1.311-7.025)$ & 0.010 \\
Baseline 6MWT & $0.995(0.992-0.998)$ & \\
\hline Distance walked, meters & $0.895(0.821-0.974)$ & 0.001 \\
\hline Lowest oxygen saturation, \% & $2.811(1.421-5.560)$ & 0.011 \\
GAP index $>5$ (stage III) & 0.003 \\
HRCT & $0.958(0.291-3.153)$ & \\
\hline Reticulations & $4.819(0.280-83.032)$ & 0.943 \\
\hline GGO & $1.273(0.880-1.839)$ & 0.279 \\
\hline Emphysema & 0.200
\end{tabular}

HR: hazard ratio; CI: confidence interval; PFTs: pulmonary function tests; FVC\%: forced vital capacity percentage predicted; DLCO: diffuse capacity for carbon monoxide percentage predicted; 6MWT: six minutes walking tests; HRCT: high resolution computed tomography; GGO: ground glass opacities. 
quency of IPAF in a cohort of patients with only NSIP and to compare the clinical features of IPAF, UCTD and iNSIP. Regarding UCTD classification, our real life data nicely fit with those of Corte et al. [11] and Nunes et al. [4], who found that $31 \%$ and $25 \%$ of histologically proven NSIP satisfied UCTD criteria. Similarly to the results presented by Ferri et al. [12], who showed clinical overlapping between IPAF and UCTD, in our study no statistically significant differences were observed in regards to age, gender, smoking habit and PFTs among IPAF, UCTD and iNSIP. IPAF and UCTD classification criteria seem to identify a population of patients with similar characteristics, although IPAF criteria are more inclusive than UCTD criteria ( $40 v s$ s $7 \%$ of NSIP patients). This finding might be explained by the fact that some clinical characteristics (e.g., mechanic's hands) and serological features (e.g., positivity of antisynthetase antibodies other than anti-Jol) are not considered classification criteria in UCTD. On the other hand, the UCTD criteria seem to be less specific than those defining IPAF. In our cohort 5 patients classified as only UCTD, but not satisfying IPAF criteria, were characterized by signs and symptoms not specific for autoimmune disorder (weight loss, gastro-oesophageal reflux and ANA/RF positivity at low title).

The overall three-year survival of our cohort of NSIP patients was $73 \%$, worse than that observed by Travis et al. $82.3 \%$ at five years and $73.2 \%$ at 10 years) [7], and, similarly to Kim et al. [13] who did not find any difference in survival between patients with UCTD and iNSIP, we found similar survival in UCTD, IPAF and iNSIP patients.

As in studies on idiopathic pulmonary fibrosis [14-16], older age and lower FVC and DLCO values were found to correlate with mortality, confirming the importance of lung function in defining the prognosis of ILDs. The correlation we observed between GAP III index and mortality confirms the results by Ryerson and colleagues [17], who demonstrated the utility of GAP index not only in Idiopathic pulmonary fibrosis but also in other ILDs.
Nineteen percent of patients in our cohort were characterized by anti-Jo1 or other antisynthetase antibodies positivity. The subjects known to have a NSIP pattern on HRCT and a positive serologic testing for antisynthetase antibodies can be classified as ASS [9] as well as IPAF [6], and this may be a confounding factor. In fact, we observed some differences between these two subgroups. The IPAF-ASS subgroup demonstrated a more acute onset (44\% vs $9 \%$ in the IPAFnonASS subgroup), similarly to the cohort by Tillie-LeBlond et al., who reported an acute onset in $47 \%$ of patients [18].

The IPAF-ASS subgroup showed a trend towards worse survival in comparison to the IPAF-nonASS subgroup (62\% vs $87 \%$ at 3 years, respectively). On the contrary, Zamora et al. observed a good overall survival (68\% at 5 years) in a large retrospective cohort of ASS [19]. This difference may be explained by the fact that Zamora and coauthors analysed only anti-Jol positive patients, while we also included patients with positivity for antisynthetase antibodies other than anti-Jol (e.g., PL7 and PL12) that, according to previous studies, seem to determine a worse survival [20-23]. According to these results, ASS seems to represent a distinct nosological entity, with specific clinical features and prognosis [24].

Limitations of the present study to be acknowledged mainly address the monocentric retrospective design. Furthermore, the enlarged antimyositis antibodies panel was not applied to all patients and, finally, we lacked lung tissue specimens to confirm the histological NSIP pattern. Furthermore, as explained above, given the paucity of study population, 23 patients overlapped in the IPAF and UCTD groups.

In conclusion, our study confirms that NSIP pattern on HRCT is often associated to autoimmunity features and that the presence of this latter characteristic does not change the outcome compared to iNSIP. IPAF criteria seem to be more inclusive than UCTD criteria in classifying this group of patients. Further studies on larger cohorts are needed in order to verify if ASS with pulmonary involvement should be included in IPAF classification.

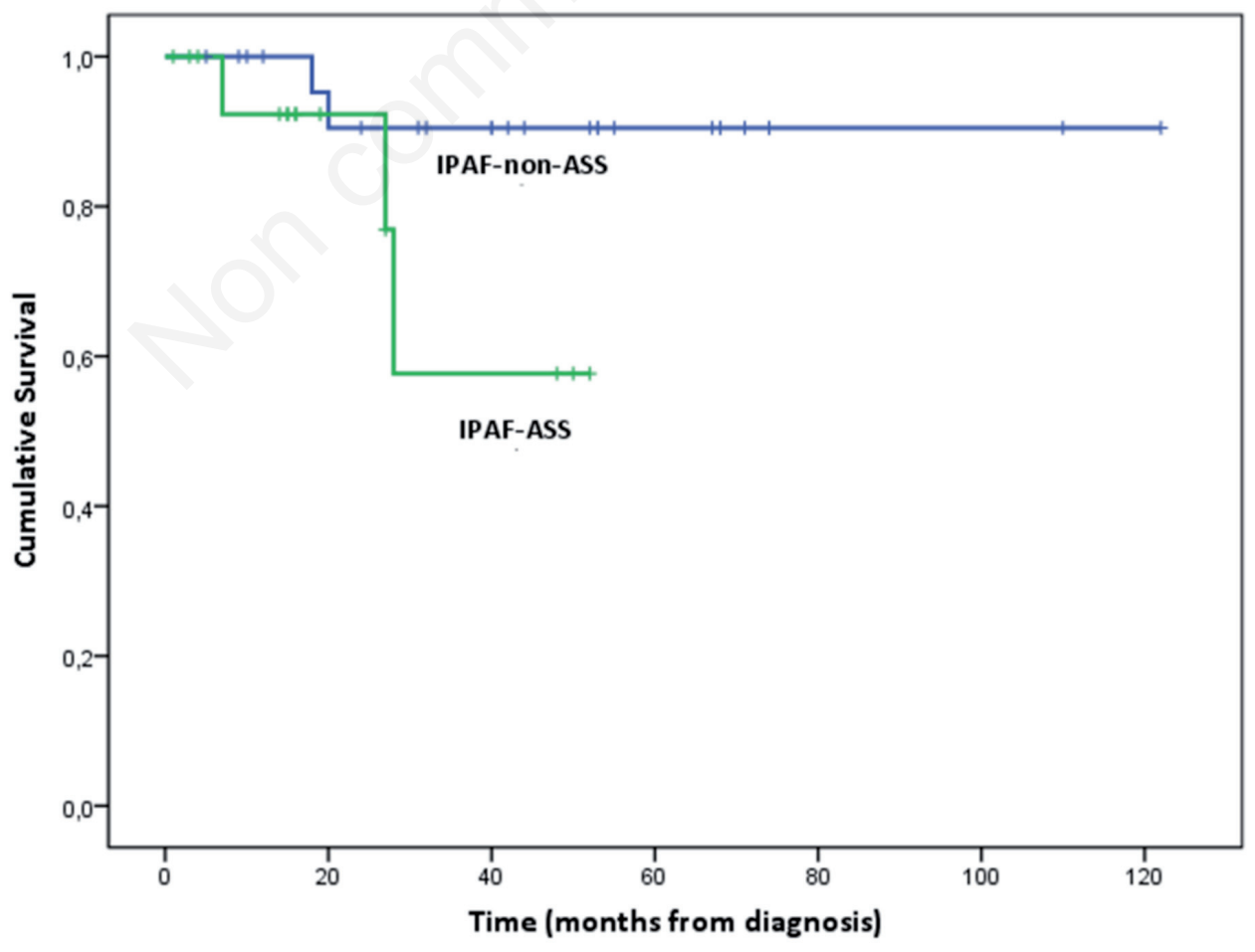

Figure 1. Kaplan Meier survival analysis comparing IPAF-ASS and IPAF-non-ASS subgroups. IPAF-ASS: IPAF patients with positive antisynthetase antibodies; IPAF-non-ASS: IPAF patients with negative antisynthetase antibody. 


\section{References}

1. Lamblin C, Bergoin C, Saelens T, Wallaert B. Interstitial lung diseases in collagen vascular diseases. Eur Respir J Suppl 2001;32:69s-80s.

2. Doria A, Mosca M, Gambari PF, Bombardieri S. Defining unclassifiable connective tissue diseases: incomplete, undifferentiated, or both? J Rheumatol 2005;32:213-5.

3. Kinder BW, Collard HR, Koth L, et al. Idiopathic nonspecific interstitial pneumonia: lung manifestation of undifferentiated connective tissue disease? Am J Respir Crit Care Med 2007;176:691-7.

4. Nunes H, Schubel K, Piver D, et al. Nonspecific interstitial pneumonia: survival is influenced by the underlying cause. Eur Respir J 2015;45:746-55.

5. Fischer A, West SG, Swigris JJ, Brown KK, du Bois RM. Connective tissue disease-associated interstitial lung disease: a call for clarification. Chest 2010;138:251-6.

6. Fischer A, Antoniou KM, Brown KK, et al. An official European Respiratory Society/American Thoracic Society research statement: interstitial pneumonia with autoimmune features. Eur Respir J 2015;46:976-87.

7. Travis WD, Hunninghake G, King TE, et al. Idiopathic nonspecific interstitial pneumonia: report of an American Thoracic Society project. Am J Respir Crit Care Med 2008;177:1338-47.

8. Silva CIS, Müller NL, Hansell DM, et al.Nonspecific interstitial pneumonia and idiopathic pulmonary fibrosis: changes in pattern and distribution of disease over time. Radiology 2008;247:251-9.

9. Connors GR, Christopher-Stine L, Oddis CV, Danoff SK. Interstitial lung disease associated with the idiopathic inflammatory myopathies: what progress has been made in the past 35 years? Chest 2010;138:1464-74.

10. Ley B, Ryerson CJ, Vittinghoff E, et al. A multidimensional index and staging system for idiopathic pulmonary fibrosis. Ann Intern Med 2012;156:684-91.

11. Corte TJ, Copley SJ, Desai SR, et al. Significance of connective tissue disease features in idiopathic interstitial pneumonia. Eur Respir J 2012;39:661-8.

12. Ferri C, Manfredi A, Sebastiani M, et al. Interstitial pneumonia with autoimmune features and undifferentiated connective tissue disease: Our interdisciplinary rheumatology-pneumology experience, and review of the literature. Autoimmun Rev 2016;15:61-70.

13. Kim H-C, Ji W, Kim MY, et al. Interstitial pneumonia related to undifferentiated connective tissue disease: pathologic pattern and prognosis. Chest 2015;147:165-72.

14. Ley B, Collard HR, King TE. Clinical course and prediction of survival in idiopathic pulmonary fibrosis. Am J Respir Crit Care Med 2011;183:431-40.

15. Raghu G, Collard HR, Egan JJ, et al. An official ATS/ERS/JRS/ALAT statement: idiopathic pulmonary fibrosis: evidence-based guidelines for diagnosis and management. Am J Respir Crit Care Med 2011;183:788-824.

16. Nathan SD, Shlobin OA, Weir N, et al. Long-term course and prognosis of idiopathic pulmonary fibrosis in the new millennium. Chest 2011;140:221-9.

17. Ryerson CJ, Vittinghoff E, Ley B, et al. Predicting survival across chronic interstitial lung disease: the ILD-GAP model. Chest 2014;145:723-8.

18. Tillie-Leblond I, Wislez M, Valeyre D, et al. Interstitial lung disease and anti-Jo-1 antibodies: difference between acute and gradual onset. Thorax 2008;63:53-9.

19. Zamora AC, Hoskote SS, Abascal-Bolado B, et al. Clinical features and outcomes of interstitial lung disease in anti-Jo-1 positive antisynthetase syndrome. Respir Med 2016;118:39-45.

20. Marie I, Josse S, Decaux 0, et al. Comparison of long-term outcome between anti-Jo1- and anti-PL7/PL12 positive patients with antisynthetase syndrome. Autoimmun Rev 2012;11:739-45.

21. Hervier B, Devilliers H, Stanciu R, et al. Hierarchical cluster and survival analyses of antisynthetase syndrome: phenotype and outcome are correlated with anti-tRNA synthetase antibody specificity. Autoimmun Rev 2012;12:210-7.

22. Hervier B, Benveniste 0. Clinical heterogeneity and outcomes of antisynthetase syndrome. Curr Rheumatol Rep 2013;15:349.

23. Aggarwal R, Cassidy E, Fertig N, et al. Patients with non-Jo-1 antitRNA-synthetase autoantibodies have worse survival than Jo-1 positive patients. Ann Rheum Dis 2014;73:227-32.

24. Monti S, Montecucco C, Cavagna L. Clinical spectrum of anti-Jo-1associated disease. Curr Opin Rheumatol 2017;29:612-7. 